\title{
Utilidad y seguridad de la biopsia estereotáctica en lesiones de fosa posterior en pacientes adultos
}

\author{
Usefulness and safety from stereotactic biopsy in posterior fossa lesions in adult patients
}

\author{
Sergio Moreno-Jiménez ${ }^{1,2 *}$, Néstor Martínez-Vaca1', Bárbara Pérez-Aguilar , Brenda Gómez-Calva1, \\ Jorge J. Díaz-Chávez y Michel G. Mondragón-Soto ${ }^{3}$ \\ 'Unidad de Radiocirugía, Instituto Nacional de Neurología y Neurocirugía; ${ }^{2}$ Clínica de Radiocirugía, Centro Neurológico del Centro Médico ABC; \\ ${ }^{3}$ Centro Médico ABC. Ciudad de México, México
}

\section{Resumen}

Antecedentes: La biopsia por estereotaxia ha sido reportada como segura y útil en pediatría. En adultos es más controvertida debido a la mayor diversidad de opciones diagnósticas. Objetivo: Demostrar su utilidad y seguridad en pacientes adultos con patología de fosa posterior. Método: En 2006-2014 se operaron 23 pacientes de fosa posterior. Variables: edad, sexo, diagnóstico preoperatorio y posoperatorio, estereotáctico, localización y complicaciones. Resultados: $52.2 \%$ mujeres y $47.8 \%$ hombres. La localización fue la región pontomesencefálica en el $43.5 \%$, el cerebelo en el 39.1\%, bulbar en el 13\% y pineal en el 4.3\%. El diagnóstico preoperatorio fue glioma de tallo en el 78.2\%, linfoma en el $8.7 \%$ y meningioma, metástasis y absceso en el $4.3 \%$ cada uno. En el $73.9 \%$ se utilizó el sistema Zamorano-Dujovni y en el 26.1\% el CRW. El diagnóstico definitivo fue astrocitoma pilocítico en el 17.4\%, astrocitoma difuso en el 13\%, respuesta inflamatoria en el 13\%, astrocitoma anaplásico en el 8.7\%, gliosis en el 8.7\%, y glioblastoma, tumor neuroectodérmico primitivo, germinoma, pineoctioma y criptococosis en el $4.3 \%$ cada uno. En el 17.4\% no hubo diagnóstico. El diagnóstico preoperatorio fue concordante en el $43.5 \%$. Hubo un déficit transitorio y un desplazamiento de uno de los pinchos en el $4.3 \%$ de los casos. En el $91.4 \%$ no hubo complicaciones. Conclusiones: Es un procedimiento útil, necesario y seguro en pacientes adultos.

PALABRAS CLAVE: Biopsia. Estereotaxia. Fosa posterior. Seguridad. Utilidad.

\begin{abstract}
Background: Stereotactic biopsy has been reported as a useful and safety procedure in pediatric patients. In adult patients exist more controversy because a greater number of diagnostic options. Objective: To demonstrate its usefulness and safety in adult patients with posterior fossa pathology. Method: From 2006-2014, 23 patients were operated from posterior fossa. Variables: age, gender, state, pre- and postoperative diagnosis, stereotactic device, location and complications. Results: $52.2 \%$ females and $47.8 \%$ males. The location was ponto-mesencephalic $43.5 \%$, cerebellum $39.1 \%$, bulbar $13 \%$ and pineal region 4.3\%. The preoperative diagnosis was brainstem glioma $78.2 \%$, lymphoma $8.7 \%$, and meningioma, metastasis and abscess 4.3\% each one. In 73.9\% Zamorano-Dujovni device was used and in $26.1 \%$ the CRW. The definitive diagnosis was pilocytic astrocytoma $17.4 \%$, diffuse astrocytoma $13 \%$, inflammatory response $13 \%$, anaplastic astrocytoma $8.7 \%$, gliosis $8.7 \%$, glioblastoma, neuroectodermic primitive tumor, germinoma, pineocytoma and cryptococcosis $4.3 \%$ each one. In $17.4 \%$ there was no diagnosis. The preoperative diagnosis was concordant in $43.5 \%$. One transient deficit and one pin displacement $4.3 \%$ were present. $91.4 \%$ without complications. Conclusions: It is a useful, necessary and safety procedure in adult patients.
\end{abstract}

KEY WORDS: Biopsy. Stereotaxy. Posterior fossa. Safety. Usefulness.

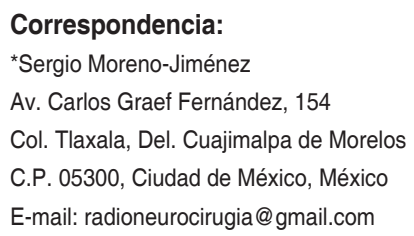

Fecha de recepción: 11-09-2018

Fecha de aceptación: 06-04-2019 DOI: $10.24875 / C I R U .19000717$
Cir Cir. 2019;87:554-558

Contents available at PubMed www.cirugiaycirujanos.com 


\section{Introducción}

A pesar del gran avance en los estudios de resonancia magnética y de medicina nuclear, estos siguen siendo insuficientes para tener información con el fin de ofrecer un tratamiento específico en todos los casos ${ }^{1}$.

La biopsia por estereotaxia es un procedimiento que ha sido utilizado para pacientes con patología benigna y maligna, pediátricos y adultos ${ }^{2,3}$.

Hay estudios que han demostrado la utilidad y la seguridad de la biopsia por estereotaxia en pacientes pediátricos con lesiones de tallo cerebral ${ }^{4}$; sin embargo, existen otros estudios en los que se ofrece un tratamiento específico con el diagnóstico por imagen de resonancia magnética sin necesidad de confirmación histopatológica, haciendo referencia a la utilidad de los estudios de imagen, específicamente la resonancia magnética, y evitar someter a los pacientes al riesgo quirúrgico ${ }^{5}$.

El objetivo de este trabajo es demostrar la utilidad de la realización de la biopsia por estereotaxia, así como su seguridad, en una población de pacientes adultos con patología de fosa posterior, ya que la mayor información de la literatura es en relación con los pacientes pediátricos debido a la mayor frecuencia de lesiones de fosa posterior en este grupo de edad. La administración de un tratamiento empírico, sin diagnóstico histopatológico, podría resultar en una respuesta fallida.

\section{Método}

Revisamos los expedientes de pacientes operados en el Instituto Nacional de Neurología y Neurocirugía en el período 2006-2014 mediante la técnica de biopsia por estereotaxia. Todos fueron operados por el mismo neurocirujano. En total fueron 129 pacientes operados, de los cuales 106 fueron supratentoriales y 23 infratentoriales. Revisamos con detalle los 23 expedientes de los pacientes sometidos a biopsia por estereotaxia de fosa posterior, tomando en cuenta la edad, el sexo, el diagnóstico preoperatorio, el diagnóstico posoperatorio, el sistema estereotáctico utilizado, la localización específica y las complicaciones.

Para la técnica de estereotaxia con un equipo Zamorano-Dujovni, después de realizar antisepsia del área frontal y occipital con jabón quirúrgico, bajo anestesia local con xilocaína simple al $2 \%$ se realiza una infiltración en el sitio de los cuatro pinchos, dos frontales justo medial a la carilla lateral del frontal en cada lado, y dos occipitales, colocando el anillo estereotáctico invertido (postes dirigidos de arriba hacia abajo), con la marca de cero grados hacia delante y abajo, cuidando de dejar suficiente espacio para que cupieran las cuatro fiduciales sin quedar forzadas, especialmente la posterior y las dos laterales. Posteriormente se lleva al tomógrafo, se fija el anillo de estereotaxia a una base universal y se realiza la adquisición de una tomografía simple y contrastada de cráneo con cortes de no más de $1 \mathrm{~mm}$. Se imprime la placa que corresponde a la localización exacta del sitio a biopsiar y se calculan manualmente las coordenadas lineales $x$ (lateromedial), $y$ (anteroposterior) y $z$ (dorsoventral). En la mayoría de los casos se planeó solo el blanco y en algunos casos se planeó también el sitio de entrada, calculando las coordenadas angulares (ángulos alfa y beta) mediante cálculos de trigonometría (alfa $=\operatorname{Tan}^{-1}$ $[\mathrm{dz} / \mathrm{dy}]$, beta $\left.=\operatorname{Tan}^{-1}[\mathrm{dx} / \mathrm{dM} 1 \mathrm{M} 2]\right)$. Una vez en la sala de operaciones, con el paciente en decúbito supino, con la mesa quirúrgica en posición de Fowler y la cabeza del paciente flexionada, se realiza asepsia del área quirúrgica y colocamos los campos estériles. Bajo anestesia local, se infiltra con xilocaína al $2 \%$ con epinefrina, y se realiza incisión y disección por planos hasta llegar a la escama del occipital, donde se realiza un trépano. Se coloca el sistema de estereotaxia y se verifica en dos ocasiones la adecuada colocación de las coordenadas $x, y$ y $z$. Se impregna la duramadre con xilocaína al $2 \%$ y se realiza coagulación e incisión (Fig. 1). Se introduce la cánula de biopsia previamente calibrada hasta llegar al blanco, en el cual se toman dos muestras de cada cuadrante, todo el tejido para estudio definitivo. Finalmente, se realiza el cierre de la manera habitual, sin dejar una burbuja de aire como marca, como en general se hace en las biopsias supratentoriales, por tratarse de la fosa posterior. Todo el tiempo se mantiene ocluida la endocánula o con solución, para evitar el neumoencéfalo, ya que la dirección de la cánula de biopsia en estos casos es de abajo arriba. El paciente se mantiene despierto o con sedación ligera durante todo el procedimiento.

Con el sistema de estereotaxia CRW la técnica es parecida, con la excepción de que el anillo se coloca de la manera habitual (no invertido) y se hace una corrección del cálculo de la coordenada $z$, a la cual se le restan $60 \mathrm{~mm}$ para normalizarla al cero estereotáctico.

En todos los casos el paciente salió de la sala de operaciones directo a un estudio de tomografía simple de cráneo y a la sala de recuperación (Figs. 2 y 3).

Para los cálculos estadísticos se realizó un análisis descriptivo con frecuencias en porcentajes, además 


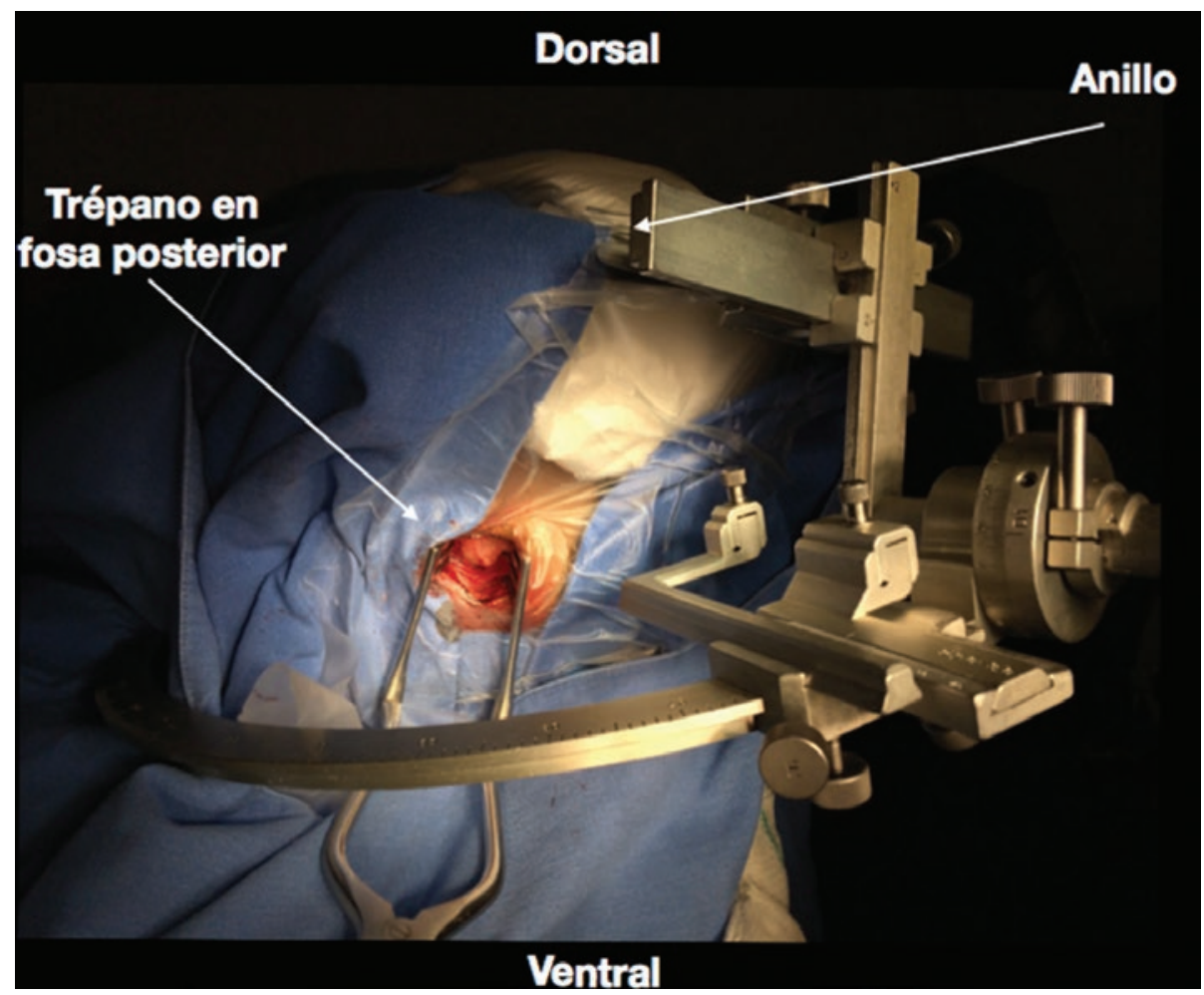

Figura 1. Montaje del sistema estereotáctico Zamorano Dujovni, con el paciente en decúbito supino, en posición de Fowler, con la cabeza flexionada, el anillo dorsal y los postes y el sistema hacia abajo del paciente.

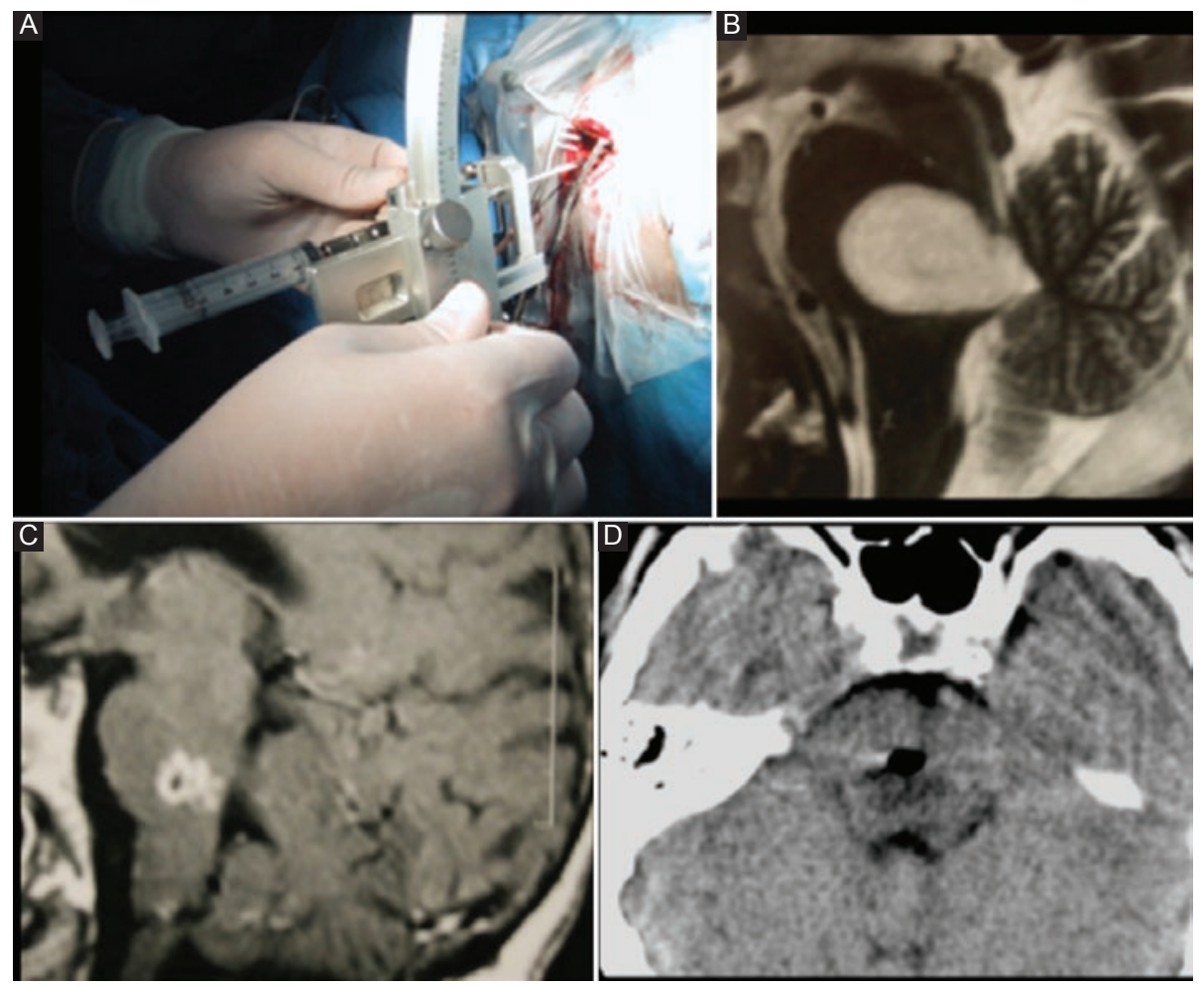

Figura 2. Se muestra uno de los casos en el cual se drenó un absceso pontino mediante biopsia por estereotaxia con el sistema ZamoranoDujovni. A: La aguja atravesando el trépano para la toma de la muestra. B: Corte sagital de una resonancia magnética de cráneo en secuencia ponderada en T2 que muestra una imagen de aspecto quístico en el puente. C: Resonancia magnética ponderada en T1 con contraste que muestra el aspecto posoperatorio de lo que fue diagnosticado como un absceso pontino. D: Tomografía simple posoperatoria inmediata que muestra el sitio de punción del drenaje del absceso. 


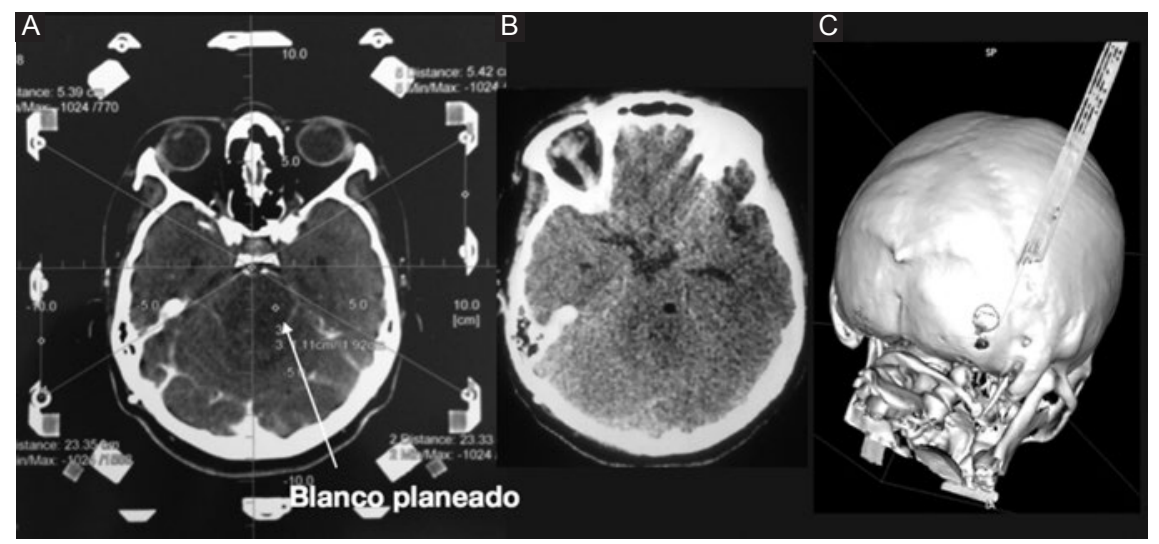

Figura 3. Se muestra otro caso de la serie que resultó tener un astrocitoma difuso. A: Tomografía contrastada de cráneo con fiduciales del sistema CRW que muestra el sitio planeado para el blanco (flecha). B: Sitio con burbuja de aire que indica el sitio de toma de biopsia. C: Reconstrucción tridimensional de una tomografía posoperatoria que muestra el trépano hacia la fosa posterior.

de medidas de tendencia central con media y desviación estándar.

\section{Resultados}

De los 23 pacientes operados de biopsia de lesiones de fosa posterior, $12(52.2 \%)$ eran mujeres y $11(47.8 \%)$ eran hombres. La media de edad fue de 43 años (rango: 15-80; desviación estándar: 16.7).

La localización de la lesión en la fosa posterior fue la región pontomesencefálica en diez (43.5\%) pacientes, el cerebelo en nueve (39.1\%), bulbar en tres (13\%) y pineal en uno (4.3\%).

El diagnóstico presuntivo según el criterio del médico tratante, tomando en cuenta los estudios de imagen y el cuadro clínico del paciente, fue glioma de tallo en $18(78.2 \%)$ pacientes, linfoma en dos (8.7\%), y meningioma, metástasis y absceso en uno $(4.3 \%)$ cada uno.

En $17(73.9 \%)$ de los 23 casos se utilizó el sistema de estereotaxia Zamorano-Dujovni, mientras que en los seis (26.1\%) restantes se usó el sistema de estereotaxia CRW.

El resultado del estudio definitivo de patología fue tumoral en el $56.3 \%$, infecciosa en el $4.3 \%$, diagnóstico descriptivo en el $21.7 \%$ y sin diagnóstico en el $17.4 \%$. De los diagnósticos tumorales, la gran mayoría fueron de estirpe astrocitaria, siendo astrocitoma pilocítico cuatro $(17.4 \%)$ casos, astrocitoma difuso tres (13\%), astrocitoma anaplásico dos (8.7\%) y glioblastoma uno (4.3\%), además de un tumor neuroectodérmico primitivo $(4.3 \%)$, un germinoma (4.3\%) y un pineocitoma (4.3\%). De patología infecciosa hubo un solo caso de criptococosis (4.3\%). Los diagnósticos descriptivos fueron respuesta inflamatoria en tres $(13 \%)$ pacientes y gliosis en dos (8.7\%), los cuales no corresponden a ningún diagnóstico preciso, por lo que se requirió realizar una biopsia abierta en dos casos y vigilancia en tres. Los cuatro pacientes sin diagnóstico fueron reoperados para biopsia abierta. Hubo nueve posibilidades diagnósticas diferentes en esta serie.

El diagnóstico preoperatorio fue correcto solo en 10 de los 23 casos (43.5\%).

De los 23 pacientes sometidos a biopsia de fosa posterior, uno $(4.3 \%)$ presentó hemorragia intratumoral de una lesión pontina que provocó un deterioro transitorio de su estado de alerta, siendo el diagnóstico final el de glioblastoma, y otro $(4.3 \%)$ presentó desplazamiento de uno de los pinchos sin mayor daño, requiriendo su recolocación y nueva planeación estereotáctica. En 21 (91.4\%) pacientes no se presentaron complicaciones.

\section{Discusión}

En pacientes pediátricos, la elevada incidencia de gliomas de tallo, así como su presentación clínica y los estudios de resonancia magnética muy sugestivos de ese diagnóstico permiten, en muchos casos, ofrecer un tratamiento sin la necesidad de hacer una confirmación histopatológica mediante toma de biopsia; sin embargo, en pacientes adultos, la mayor diversidad de diagnósticos en esta localización hace que la biopsia sea de utilidad ${ }^{6}$. En nuestra serie tuvimos nueve posibilidades diagnósticas diferentes, entre diagnósticos precisos tumorales o infecciosos, o diagnósticos descriptivos. Cabe mencionar que hubo un paciente con glioblastoma, el cual es extremadamente raro en la fosa posterior.

En los pacientes pediátricos se han sugerido tres indicaciones para tomar una biopsia: la presencia de 
una masa con reforzamiento con contraste peripontina, mesencefálica o bulbar, o un tumor exofítico hacia el cuarto ventrículo, un diagnóstico dudoso, o cuando el resultado permita confirmar el diagnóstico y permitir que el paciente entre a un protocolo de investigación. Estas características se presentan en aproximadamente el $10-15 \%$ de todos los casos ${ }^{7}$.

Los estudios de resonancia magnética tienen limitaciones para determinar el diagnóstico correcto en pacientes con lesiones del tallo cerebral, y logran un diagnóstico correcto solo en el $35 \%$ de los casos de patología de bajo grado y en el $27 \%$ de los de patología de alto grado de malignidad en adultos ${ }^{8}$. En nuestra serie, la determinación correcta del diagnóstico con el uso solo de estudios de imagen fue del $43.5 \%$ (10 de 23 casos). Esta asertividad, mayor que la reportada en la literatura, pudiera deberse a la mejoría en la calidad de los estudios de neuroimagen con los que se cuenta en la actualidad, pero continúa siendo baja, haciendo notar la necesidad de realizar una biopsia por estereotaxia para confirmar el diagnóstico y poder ofrecer un tratamiento específico.

Se han descrito diversas técnicas para tomar biopsias por estereotaxia en lesiones de fosa posterior. Se ha utilizado el sistema de Leksell con un abordaje transcerebeloso con el anillo en la posición habitual ${ }^{9}$ 0 con el anillo invertido ${ }^{10}$. En nuestra serie utilizamos el anillo invertido con el sistema Zamorano-Dujovni en el $73.9 \%$ de los casos, y en posición habitual con el sistema CRW en el $26.1 \%$.

Un metaanálisis de 735 casos de pacientes pediátricos con tumores de tallo cerebral biopsiados por estereotaxia concluyó que la morbilidad permanente general es del $0.6 \%$ (intervalo de confianza del $95 \%$ : $0.2 \%-1.4 \%$ ), sin encontrar diferencia en los resultados comparando la trayectoria transfrontal con la transcerebelosa ${ }^{11}$. En nuestra serie no tuvimos pacientes con morbilidad permanente.

En una serie de 82 pacientes se reportaron dos hematomas asintomáticos (2.4\%), cinco deterioros transitorios $(6.1 \%)$ y solo dos deterioros permanentes $(2.4 \%)^{12}$. En nuestra serie tuvimos un hematoma con deterioro neurológico transitorio $(4.3 \%)$ y un deslizamiento del pincho sin mayor repercusión (4.3\%), lo cual se encuentra dentro de lo reportado en la literatura.

\section{Conclusiones}

La biopsia por estereotaxia de lesiones de fosa posterior es un procedimiento de bajo riesgo, además de necesario para lograr un diagnóstico de certeza posibilidades diagnósticas. Cabe mencionar que se obtuvo un diagnóstico de certeza en el $61 \%$ de los casos.

\section{Conflicto de intereses}

Los autores hacemos constar que no tenemos conflicto de intereses, además de que no contamos con ningún tipo de financiamiento.

\section{Responsabilidades éticas}

Protección de personas y animales. Los autores declaran que para esta investigación no se han realizado experimentos en seres humanos ni en animales.

Confidencialidad de los datos. Los autores declaran que han seguido los protocolos de su centro de trabajo sobre la publicación de datos de pacientes.

\section{Derecho a la privacidad y consentimiento infor-} mado. Los autores han obtenido el consentimiento informado de los pacientes y/o sujetos referidos en el artículo. Este documento obra en poder del autor de correspondencia.

\section{Bibliografía}

1. Massager N, David P, Goldman S, Pirotte B, Wikler D, Salmon I, et al. Combined magnetic resonance imaging and positron emission tomography guided sterotactic biopsy in brainstem mass lesions: diagnostic yield in a series of 30 patients. J Neurosurg. 2000;93:951-7.

2. Beynon C, Neuman JO, Bösel J, Unterberg AW, Kiening KL. Stereotactic biopsy and drainage of brainstem abscess caused by Listeria monocytogenes. Neurol Med Chir (Tokyo). 2013;53:263-5.

3. Goncalves-Ferreira AJ, Herculano-Carvalho M, Pimentel J. Stereotactic biopsies of focal brainstem lesions. Surg Neurol. 2003;60:311-20.

4. Cage TA, Samagh SP, Mueller S, Nicolaides T, Haas-Kogan D, Prados M, et al. Feasability, safety, and indications for surgical biopsy of intrinsic brainstem tumors in children. Childs Nerv Syst. 2013;29:1313-9.

5. Marcus KJ, Dutton SC, Barnes P, Coelman CN, Pomery SL, Goumnerova L, et al. A phase I trial of etanidazole and hyperfractionated radiotherapy in children with diffuse brainstem glioma. Int J Radiat Oncol Biol Phys. 2003;55:1182-5.

6. Samadani U, Stein S, Moonis G, Sonnad SS, Bonura P, Judy KD. Stereotactic biopsy of brain stem masses: decision analysis and literature review. Surg Neurol. 2006;6:484-91.

7. Albright AL, Packer RJ, Zimmerman R, Rorke LB, Boyett R, Hammond GD. Magnetic resonance scans should replace biopsies for the diagnosis of diffuse brain steam gliomas. Neurosurgery. 1993;33:1026.

8. Rachinger W, Grau S, Holtmannspötter M, Herms J, Tonn JC, Kreth FW. Serial stereotactic biopsy of brainstem lesions in adults improves diagnostic accuracy compared with MRI only. J Neurol Neurosurg Psychiatry. 2009;80:1134-9

9. Neal J, Van Norman AS. Transcerebellar biopsy of posterior fossa lesions using the Leksell Gama Model stereotactic frame. Neurosurgery. 1993;32:1992-8

10. Capitanio JF, Camporesi S, Franzin A, Barzaghi LR, Picozzi P, Mortini P. Inverted positioning of Leksell frame $G$ for very low posterior fossa and brain stem lesions biopsies. J Neurosurg Sci. 2016;15:1-6.

11. Hamish C, Kickingereder P, Fischer M, Simon T, Ruge MI. Update on the diagnostic value and safty of stereotactic biopsy for pediatric brainstem tumors: a systemic review and meta-analysis of 735 cases. JNS Pediatrics. 2017;16:1-8.

12. Manoj N, Arivazhagan DI, Bhat DI, Arvinda HR, Mahadevan A, Santosh V, et al. Stereotactic biopsy of brainstem lesions: techniques, efficacy, safety, and disease variation between adults and children: a single institutional series and review. Journal of Neurosci Rural Pract. 2014;5:32-40. 